\title{
Sonographic Maturation of Third-Trimester Cerebellar Foliation after Birth
}

\author{
ALEX KORSTEN, MAARTEN LEQUIN, AND PAUL GOVAERT \\ Department of Neonatology [A.K., P.G.], Department of Pediatric Radiology [M.L.], Erasmus MC Sophia Children's Hospital, \\ 3015 GJ Rotterdam, The Netherlands
}

\begin{abstract}
The development of cerebellar folia of third-trimester preterms has not been described with ultrasound before. We set out to determine normal development of the pons and cerebellar folia for future measurements of hypoplasia and atrophy. Study sonograms were made in preterms admitted to the neonatal intensive care unit with postmenstrual age (PMA) from $25 \mathrm{wk}$ until term. On a weekly basis, transcranial measurements were made in the axial and coronal planes at the asterion with high-frequency transducers (8.5 and $13 \mathrm{MHz}$ ). The axial images showed the pons and fourth ventricle. The coronal images showed cerebellar folia and white matter. In the same coronal plane, the depth of cerebellar fissures at the rostral cerebellar convexity could be observed. A total of 172 cranial sonograms were performed on 98 neonates. The development of folia could be measured with ultrasound through the asterion. We showed that pons area, mean of three fissure depths, hemisphere area, and number of folia in the horizontal fissure correlated significantly with PMA. Hemisphere area described the same trend of growth as the transverse cerebellar diameter used in fetal sonography. (Pediatr Res 59: 695-699, 2006)
\end{abstract}

$\mathrm{T}$ The development of the cerebellum has been studied extensively in different fields of research. It was studied histologically by Hochstetter in 1929 (1). Ten Donkelaar et al. (2) reviewed morphogenesis and histogenesis of the cerebellum and its developmental disorders. Normative data of development of folia vermis were reported by Loeser et al. (3). A morphologic analysis of the layers of cortex cerebelli was made by Gadson and Emery (4). Imaging techniques have also been used to study cerebellar development. Normal development of the fetal vermis cerebelli can be measured using transvaginal sonography (5). TCD can be measured with ultrasound and used to determine duration of gestation and intrauterine growth restriction $(6,7)$. Normal values for TCD have also been established postmortem (8). Ultrasound imaging through the posterolateral fontanelle adds benefit to routine neonatal cranial sonography (9). Magnetic resonance imaging (MRI) studies of the cerebellum have been used for staging of cerebellar development and measurements of pons and cerebellum of preterms $(10,11)$. The morphologic development of folia, however, has not been studied with MRI or ultrasound. The aim of this study was to develop a method to

Received April 11, 2005; accepted January 9, 2006.

Correspondence: Paul Govaert, M.D., Ph.D., Department of Neonatology, Erasmus MC Sophia Children's Hospital, Rotterdam, Dr Molewaterplein 60, 3015 GJ Rotterdam, Zuid-Holland, The Netherlands; e-mail: paul.govaert3@pandora.be

DOI: 10.1203/01.pdr.0000214991.07965.0f measure third-trimester foliation through the asterion with high-frequency ultrasound.

\section{SUBJECTS AND METHODS}

Preterm neonates with gestational age ranging from $25 \mathrm{wk}$ until term admitted to the neonatal intensive care unit (NICU) were included in this study. Preterms with an infant flow system were excluded because the required bonnet and nasal mask prevent sonographic measurements at the asterion. This is the only group of neonates that was not included; all of the other neonates were included to obtain a diverse population. Because of the high turnover of neonates, a longitudinal study was not possible, so this was essentially a cross-sectional study.

On a weekly basis, sonographic measurements were made transcranially using an Acuson Sequoia 512 Ultrasound System (Siemens Medical Solutions, Malvern, PA). These measurements were performed immediately after routine examination and, therefore, no informed consent was necessary. Sonographic images were obtained with high-frequency transducers ( 8.5 and $13 \mathrm{MHz}$ ) placed at the asterion in the coronal and axial plane. The asterion is the point of meeting of the lambdoidal, masto-occipital, and mastoparietal sutures. It lies $4 \mathrm{~cm}$ behind and $12 \mathrm{~mm}$ above the level of the auricular point in adults (12). The measurements were made on the presenting side of the head. The PA was measured first. The $8.5-\mathrm{MHz}$ transducer was placed above the ear in the axial plane of the pons. To guarantee reproducibility, the plane of interest was chosen when the prepontine cistern and fourth ventricle were visible, respectively, anterior and posterior to the pons (Fig. 1A). The ellipse tool was used to measure PA. The second measurement was made with a 13-MHz transducer in the coronal plane of the cerebellum. The image obtained in this way showed cortex and white matter of one cerebellar hemisphere. The direction of the plane was chosen where the fourth ventricle has the shape of an isosceles triangle. The craniocaudal distance (CCD4) and the width (W4) of the fourth ventricle were measured (Fig. 1C). Fissures with their folia could be distinguished in the cortex. The depths of the three largest fissures were measured at the cranial convexity (Fig. 1C). MFID was used for statistical analysis. Next, a "free trace tool" was used to measure the HA (Fig. $1 D$ ). In the same coronal plane, the NFOF was counted (Fig. 1, $E$ and $G$ ). The depth of the image was set at $20 \mathrm{~mm}$ and the contrast level was set to maximum for better discrimination of the folia. The folia can be recognized as white spots, resembling apples on a tree (Fig. 1, $F$ and $H$ ). Only large dots were counted. These dots probably represent the interface between adjacent folia. The transducer was then rotated to obtain an axial image of the surface of the inferior part of the cerebellum. This way the number of folia at the surface (NFOS) of the cerebellum can be counted over a fixed distance (Fig. $1 B)$.

The data were analyzed with SPSS software (SPSS, Inc., Chicago, IL). Linear regression analysis was performed and Pearson's correlation coefficient was calculated.

\section{RESULTS}

A total of 172 cranial sonograms were performed on 98 neonates. The number of follow-up scans ranged from 1 to 3 ,

Abbreviations: HA, hemisphere area; MFID, mean of three fissure depths; NFOF, number of folia in the horizontal fissure; PA, pons area; PMA, postmenstrual age; PVL, periventricular leukomalacia; TCD, transverse cerebellar diameter 


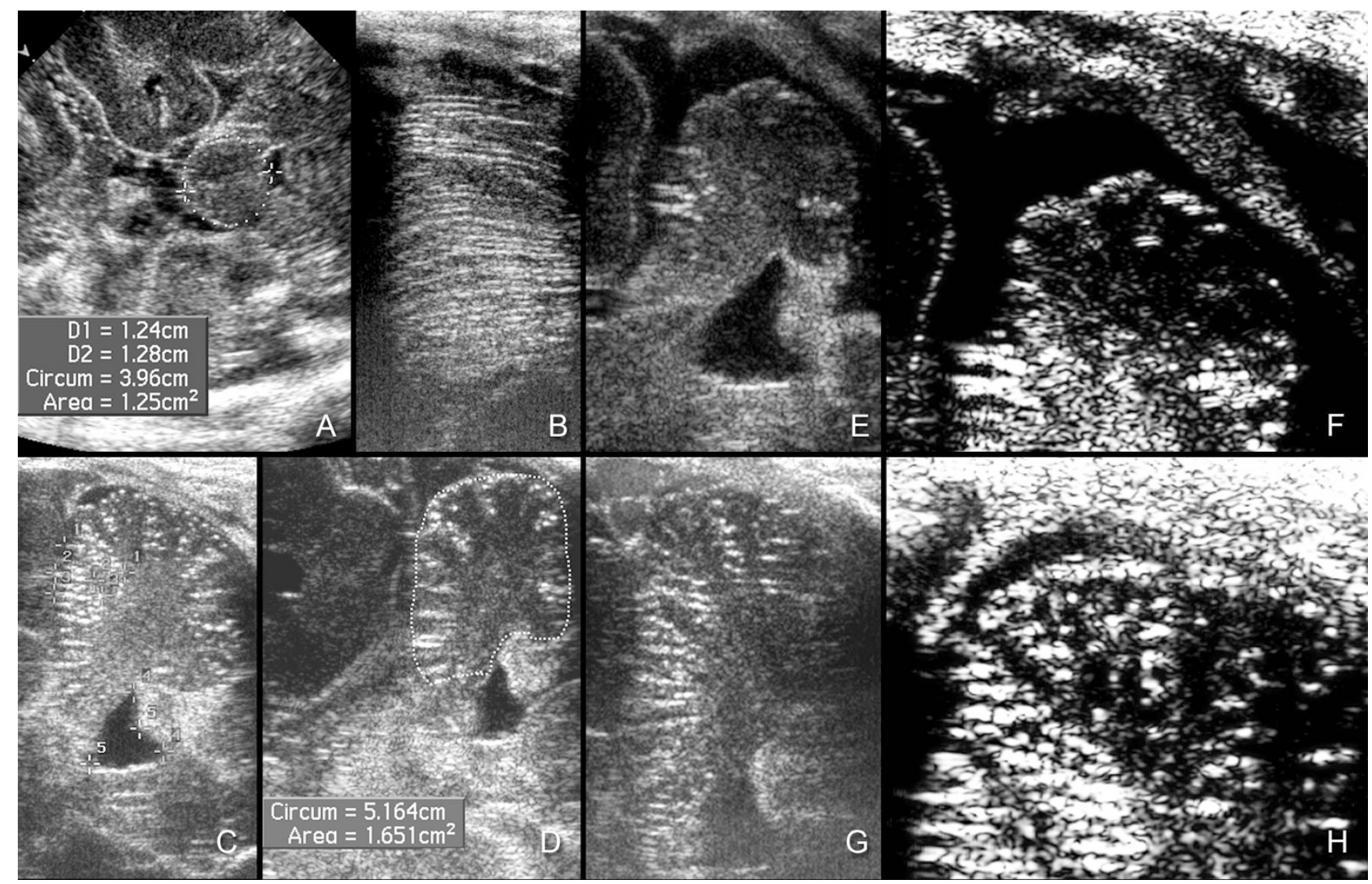

Figure 1. (A) Echo of PA; $(B)$ echo in oblique plane of section through inferior cerebellar surface at 40 wk PMA; $(C)$ echo of fissure depth; $(D)$ echo of HA; $(E)$ echo of a preterm's hemisphere at 26 wk PMA; $(F)$ NFOF at 26 wk PMA; $(G)$ echo at 42 wk PMA; $(H)$ NFOF at 42 wk PMA. The cranial side of the head is to the left and lateral side is on the top of each echo image, except in $A$, where anterior is to the left.

depending on the duration of admission at the NICU. Gestational age at birth in this patient population ranged from $252 / 7$ to 42 wk. PMA at scanning ranged from $254 / 7$ to $441 / 7$ wk. Most measurements were performed on neonates between 27 and 34 wk PMA, as shown in Figure 2A. Birth weight ranged from 635 to $4525 \mathrm{~g}$. A summary and analysis of measurements are given in Table 1. CCD4 and W4 did not correlate with PMA. A halfway evaluation of the measurements showed that NFOS was not a promising method to measure third-trimester development of folia. Hence, we developed the concept of NFOF. This measurement was then added to the sonogram and NFOS was removed. The missing measurements of PA (9), HA (11), and MFID (4) were due to incomplete digital data storage. The linear regression equations in Table 1 were calculated and described the relationships between the measured data and PMA. Figure 2, $B-F$, shows the relationships of PA, MFID, HA, NFOF, and NFOS with PMA in a graph. PA, MFID, HA, and NFOF correlated positively with PMA. NFOS correlated negatively with PMA. Data analysis showed that MFID and HA were significant for a quadratic fit, contrary to the linear fit for PA, NFOF, and NFOS. Table 1 shows the correlations between HA, MFID, PA, NFOF, and NFOS. HA correlated significantly with MFID $(r=0.792, p<0.01)$, PA $(r=0.732, p<0.01)$, and NFOF $(r=0.712, p<0.01)$.

\section{DISCUSSION}

This study demonstrates that sonographic examination of the posterior fossa through the asterion can be used to measure
PA, HA, MFID, NFOF, and NFOS. Some of these measurements are directly related to maturation of cerebellar folia. All of the measurements with the exception of NFOS relate significantly to PMA. Most measurements were performed on neonates between 27 and 34 wk PMA. We therefore assume our measurements are most reliable between 27 and 34 wk. Further studies should focus on neonates older than 34 wk or younger than 27 wk.

PA in a sagittal section through the fourth ventricle has not been measured with ultrasound before. PA measurements with MRI were performed by Argyropoulou et al. (11). That report describes the relationship between PVL and size of pons and cerebellum. In patients with PVL, the corpus callosum (used as a marker for assessment of the extent of white matter injury), pons, and cerebellum were smaller than in controls. A positive correlation was found between corpus callosum, pons, and vermis in preterms and a control group. The cerebellum only correlated with the corpus callosum in the control group. PVL is a common finding during neurosonography of preterm infants $(13,14)$. Measurements of PA may add to the sonographic study of PVL.

TCD is one way of measuring development of the cerebellum $(8,15-17)$. TCD consists of the width of two hemispheres and the vermis. We have measured the area of one hemisphere (HA), because this can be readily done without repositioning a sick preterm infant. HA turned out to be a reproducible method to follow hemisphere growth $(r=0.890, p<0.05)$. Comparing our measurements of HA with the measurements 

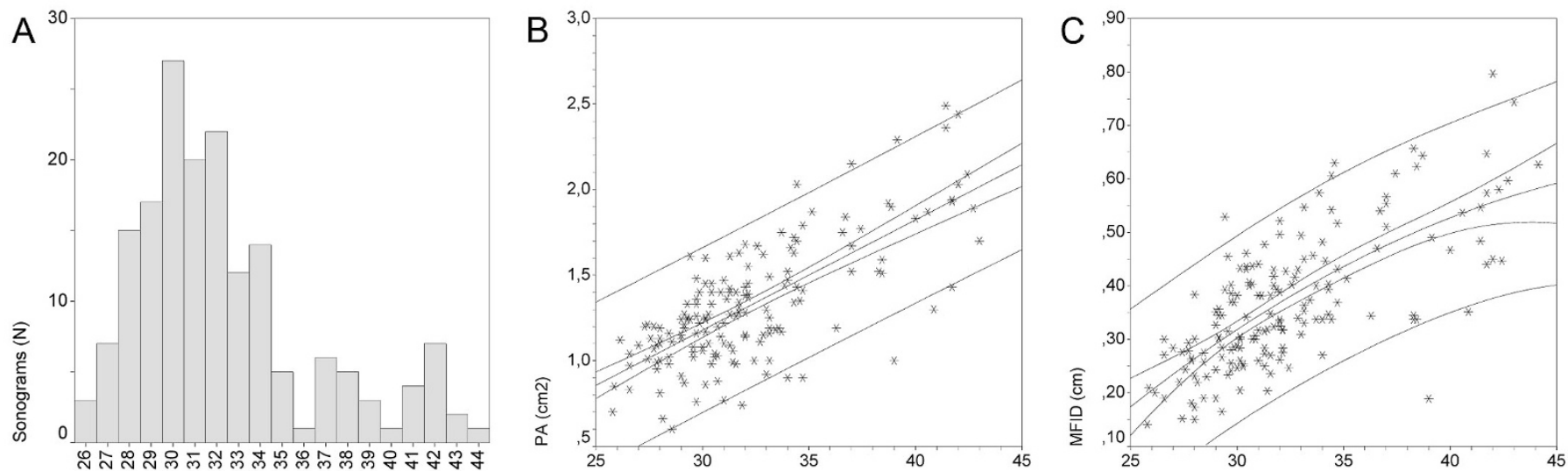

PMA (weeks)
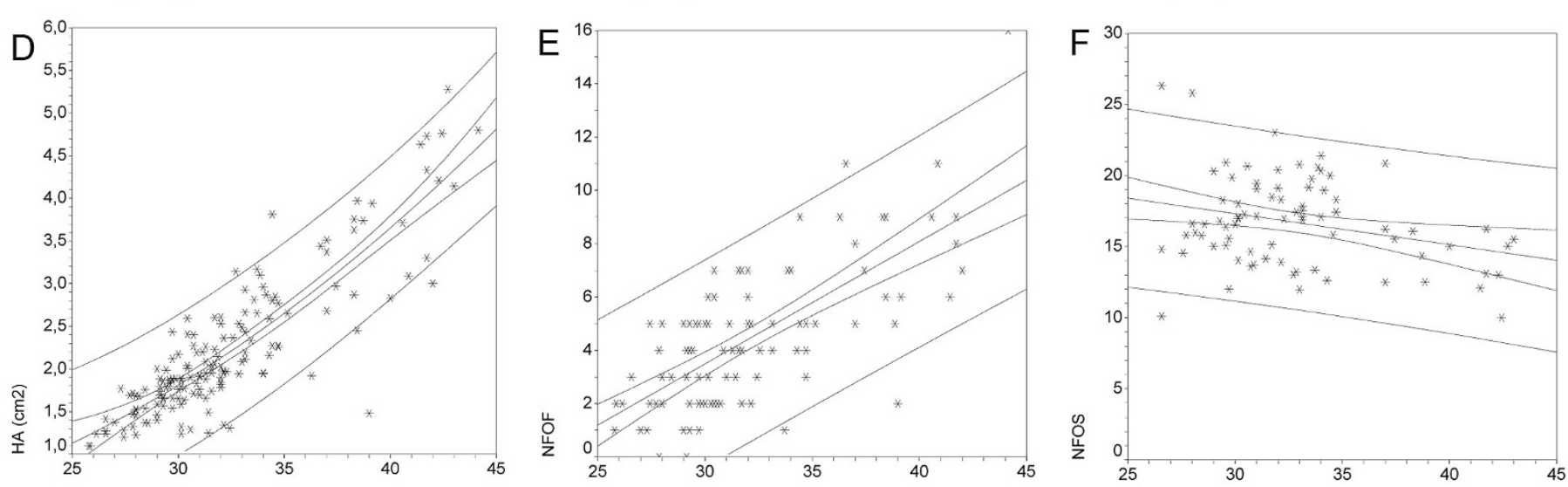

PMA (weeks)

PMA (weeks)

PMA (weeks)

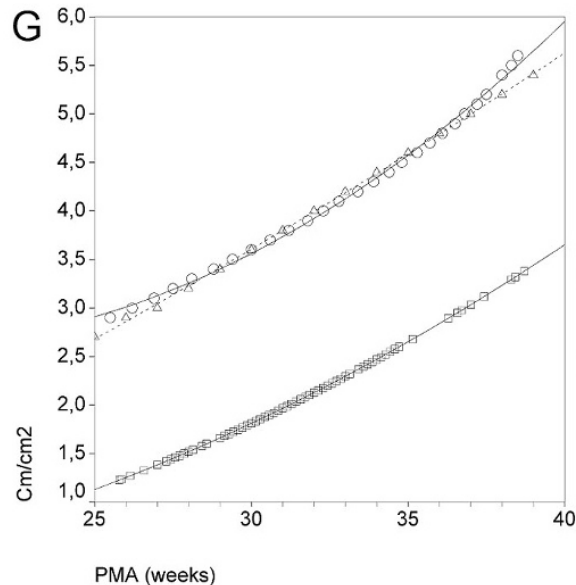

$$
\begin{aligned}
& \text { TCD with } \\
& \text { PMA }(\mathrm{cm}) \text { Pinar } 2002 \\
& \text { Predicted TCA with } \\
& \text { PMA }(\mathrm{cm}) \text { Hill } 1990 \\
& \text { Quadratic fit of HA } \\
& \text { with PMA (cm2) }
\end{aligned}
$$

Figure 2. (A) Distribution of number of sonograms with PMA. (B) Linear fit of regression for PA with PMA. (C) Quadratic fit of regression for MFID with PMA. (D) Quadratic fit of regression for HA with PMA. $(E)$ Linear fit of regression for NFOF with PMA. $(F)$ Linear fit of regression for NFOS with PMA. $(G)$ Correlation of TCD and HA with PMA, TCD $(\mathrm{cm})$ with PMA (8), predicted TCD (cm) with PMA (18), and quadratic fit for HA (cm²) with PMA. The 95\% confidence intervals are shown for individual (outer lines) and for group mean measurements.

of TCD by Hill et al. (18) and Pinar et al. (8), we can observe an identical trend of growth (Fig. $2 G$ ).

MFID was chosen as a reproducible method to appreciate the depth of the secondary fissures at the rostral cerebellar hemisphere convexity. The depth of major fissures of vermis cerebelli has been related by Makori et al. (19) to exposure to 13-cis-retinoic acid (cRA). Table 2 gives an overview of toxins that have the potential to disturb cerebellar foliation. MFID turned out to be a reproducible method $(r=0.717, p<$
0.01). In future studies, MFID may be used to measure the affect of toxins on cerebellar foliation.

Hochstetter (1) studied the growth of folia vermis in sagittal histologic sections. The sections show the increase in number of folia from 12 to 28 wk PMA. Loeser et al. (3) reported the growth of folia for each lobule from 24 to 37 wk PMA. Our measurements using ultrasonographic inspection of the maturation of the folial pattern (NFOF) confirm this increase in number of folia with increase in PMA. NFOS shows a nega- 
Table 1. Summary and analysis of measurements

\begin{tabular}{|c|c|c|c|c|c|c|c|c|}
\hline Measurement & No. of measurements & Mean & Range & \multicolumn{2}{|c|}{ SD } & $r$ & \multicolumn{2}{|c|}{ SE of the estimate } \\
\hline PA & 163 & $1.32 \mathrm{~cm}^{2}$ & $0.60-2.49 \mathrm{~cm}^{2}$ & \multicolumn{2}{|c|}{0.35} & $0.748 * *$ & \multicolumn{2}{|c|}{0.235} \\
\hline MFID & 168 & $0.37 \mathrm{~cm}$ & $0.14-0.80 \mathrm{~cm}$ & \multicolumn{2}{|c|}{0.13} & $0.717 * *$ & \multicolumn{2}{|c|}{0.104} \\
\hline HA & 161 & $2.23 \mathrm{~cm}^{2}$ & $1.10-5.28 \mathrm{~cm}^{2}$ & \multicolumn{2}{|c|}{0.84} & $0.890 *$ & \multicolumn{2}{|c|}{0.384} \\
\hline NFOS & 79 & 16.7 & $10-26$ & \multicolumn{2}{|c|}{3.17} & $0.285^{*}$ & \multicolumn{2}{|c|}{3.06} \\
\hline \multicolumn{3}{|c|}{ Linear regression equations for PMA } & Correlation & PA & MFID & HA & NFOF & NFOS \\
\hline \multirow{3}{*}{\multicolumn{3}{|c|}{$\begin{array}{l}\text { PA }\left(\mathrm{cm}^{2}\right)=0.06662 \times \text { PMA }-0.821 \\
\text { MFID }(\mathrm{cm})=-0.000546 \times \mathrm{PMA}^{2}+0.060015 \times \text { PMA }-0.991365 \\
\text { HA }\left(\mathrm{cm}^{2}\right)=0.003188 \times \mathrm{PMA}^{2}-0.033557 \times \text { PMA }-0.053502 \\
\text { NFOF }=0.486 \times \text { PMA }-11.085 \\
\text { NFOS }=-0.219 \times \text { PMA }+23.870\end{array}$}} & HA & & & 1 & $0.712 * *$ & $-0.298 * *$ \\
\hline & & & NFOF & & & & 1 & -0.232 \\
\hline & & & NFOS & & & & & 1 \\
\hline
\end{tabular}

$* p<0.05, * * p<0.01$.

Table 2. Overview of toxins that affect cerebellar folia

\begin{tabular}{|c|c|c|c|}
\hline Toxin & Place of effect & Cytotoxic effect & Reference \\
\hline Cis-dichlorodi-ammineplatinum & Purkinje neurons & $\begin{array}{l}\text { Affected morphology of folia on } \\
\text { lobules V-VII of cerebellar } \\
\text { vermis }\end{array}$ & (24) \\
\hline $\mathrm{X}$-irradiation & $\begin{array}{l}\text { Purkinje cells, Bergman glial } \\
\text { fibers }\end{array}$ & $\begin{array}{l}\text { Impaired foliation of the } \\
\text { cerebellum }\end{array}$ & (29) \\
\hline Chloro-organic pesticides & & $\begin{array}{l}\text { Retarded differentiation of } \\
\text { the nervous structure of } \\
\text { cerebellum }\end{array}$ & (30) \\
\hline
\end{tabular}

tive relation with PMA, but this is expected as with an increase in size of the folia, the number of folia over a fixed distance will decrease. NFOF and NFOS are probably not suited for comparison between individual patients because we expect limited interobserver reproducibility. Improved ultrasound imaging techniques may, in the future, produce images with greater detail and could result in a more reproducible method for counting folia. NFOF and NFOS as used in this report may be used to study cohorts of infants with bronchopulmonary dysplasia or fetal growth retardation. A comparison of NFOF and NFOS can be made between a group of preterms with, for instance, intrauterine growth retardation or chronic lung disease-disorders with chronic hypoxia that might influence development of the external and internal granular layers - and a control group (20-22).

An MRI study of preterm infants by Lin et al. (23) showed the ratio of thalami area to the cerebellum area was significantly smaller in infants with moderate and severe PVL than in the control group. Also, the ratio of cerebral hemisphere area to the cerebellum area was smaller in infants with severe PVL than in the control group. A ratio of NFOF and NFOS with cerebral hemisphere area and thalami area may be determined with ultrasound imaging in a group of preterms with PVL and a control group.
In conclusion, maturation of folia can be measured with ultrasound through the asterion in preterm infants. We showed that PA, MFID, HA, and NFOF correlate significantly with PMA. HA described the same trend of growth as TCD. Further cohort studies should provide evidence for the use of this method for recognizing impaired cerebellar foliation. Insonation via the asterion may prove useful for the description of malformations of the cerebellum presenting in the neonatal period.

Acknowledgments. The authors thank Ir. W.C.J. Hop for assistance with the statistical analysis and Prof. dr. C.I. de Zeeuw for his thoughts on the foliation of the cerebellum.

\section{REFERENCES}

1. Hochstetter F 1929 Final Delivery: The Development of Mes- and Rhombencephalon. Contribution to the History of Development of the Human Brain. Franz Deuticke, Vienna, Austria, and Leipzig, Germany, pp123-134

2. ten Donkelaar HJ, Lammens M, Wesseling P, Thijssen HO, Renier WO 2003 Development and developmental disorders of the human cerebellum. J Neurol 250:1025-1036

3. Loeser JD, Lemire RJ, Alvord EC Jr., 1972 The development of the folia in the human cerebellar vermis. Anat Rec 173:109-113

4. Gadson DR, Emery JL 1976 Some quantitative morphological aspects of post-natal human cerebellar growth. J Neurol Sci 29:137-148

5. Malinger G, Ginath S, Lerman-Sagie T, Watemberg N, Lev D, Glezerman M 2001 The fetal cerebellar vermis: normal development as shown by transvaginal ultrasound. Prenat Diagn 21:687-692 
6. Serhatlioglu S, Kocakoc E, Kiris A, Sapmaz E, Boztosun Y, Bozgeyik Z 2003 Sonographic measurement of the fetal cerebellum, cisterna magna, and cavum septum pellucidum in normal fetuses in the second and third trimesters of pregnancy. J Clin Ultrasound 31:194-200

7. Tongsong $\mathrm{T}$, Wanapirak C, Thongpadungroj $\mathrm{T} 1999$ Sonographic diagnosis of intrauterine growth restriction (IUGR) by fetal transverse cerebellar diameter (TCD)/ abdominal circumference (AC) ratio. Int J Gynaecol Obstet 66:1-5

8. Pinar H, Burke SH, Huang CW, Singer DB, Sung CJ 2002 Reference values for transverse cerebellar diameter throughout gestation. Pediatr Dev Pathol 5:489-494

9. Luna JA, Goldstein RB 2000 Sonographic visualization of neonatal posterior fossa abnormalities through the posterolateral fontanelle. AJR Am J Roentgenol 174:561567

10. Stricker T, Martin E, Boesch C 1990 Development of the human cerebellum observed with high-field-strength MR imaging. Radiology 177:431-435

11. Argyropoulou MI, Xydis V, Drougia A, Argyropoulou PI, Tzoufi M, Bassounas A, Andronikou S, Efremidis SC 2003 MRI measurements of the pons and cerebellum in children born preterm; associations with the severity of periventricular leukomalacia and perinatal risk factors. Neuroradiology 45:730-734

12. Gray H, Williams PL 1989 Gray's Anatomy. Churchill Livingstone, Edinburgh, p 1598

13. Coley BD, Hogan MJ 1997 Cystic periventricular leukomalacia of the corpus callosum. Pediatr Radiol 27:583-585

14. Grant EG, Schellinger D 1985 Sonography of neonatal periventricular leukomalacia: recent experience with a 7.5-MHz scanner. AJNR Am J Neuroradiol 6:781-785

15. Hashimoto K, Shimizu T, Shimoya K, Kanzaki T, Clapp JF, Murata Y 2001 Fetal cerebellum: US appearance with advancing gestational age. Radiology 221:70-74

16. Goldstein I, Reece EA, Pilu G, Bovicelli L, Hobbins JC 1987 Cerebellar measurements with ultrasonography in the evaluation of fetal growth and development. Am J Obstet Gynecol 156:1065-1069

17. Meyer WJ, Gauthier DW, Goldenberg B, Santolaya J, Sipos J, Cattledge F 1993 The fetal transverse cerebellar diameter/abdominal circumference ratio: a gestational age-independent method of assessing fetal size. J Ultrasound Med 12:379-382

18. Hill LM, Guzick D, Fries J, Hixson J, Rivello D 1990 The transverse cerebellar diameter in estimating gestational age in the large for gestational age fetus. Obstet Gynecol 75:981-985
19. Makori N, Peterson PE, Hendrickx AG 2001 13-cis-retinoic acid causes patterning defects in the early embryonic rostral hindbrain and abnormal development of the cerebellum in the macaque. Teratology 63:65-76

20. Le Strange E, Saeed N, Cowan FM, Edwards AD, Rutherford MA 2004 MR imaging quantification of cerebellar growth following hypoxic-ischemic injury to the neonatal brain. AJNR Am J Neuroradiol 25:463-468

21. Tsuru A, Mizuguchi M, Takashima S 1995 Cystic leukomalacia in the cerebellar folia of premature infants. Acta Neuropathol (Berl) 90:400-402

22. Mancini L, Bertossi M, Roncali L 1985 [Differentiation of cerebellar Purkinje neurons of the normal and chronically hypoxic chick embryo]. Boll Soc Ital Biol Sper 61:79-85

23. Lin Y, Okumura A, Hayakawa F, Kato K, Kuno T, Watanabe K 2001 Quantitative evaluation of thalami and basal ganglia in infants with periventricular leukomalacia. Dev Med Child Neurol 43:481-485

24. Bernocchi G, Scherini E, Nano R 1990 Developmental patterns in the rat cerebellum after cis-dichlorodiammineplatinum treatment. Neuroscience 39:179-188

25. Hipwell E, Giles J, Bannigan J 2003 Effects of ethanol on development of the external germinal layer of the mid-gestation chick cerebellum. Reprod Toxicol 17:494-495(abstr)

26. Sakata-Haga H, Sawada K, Hisano S, Fukui Y 2001 Abnormalities of cerebellar foliation in rats prenatally exposed to ethanol. Acta Neuropathol (Berl) 102:36-40

27. de la Monte SM, Wands JR 2002 Chronic gestational exposure to ethanol impairs insulin-stimulated survival and mitochondrial function in cerebellar neurons. Cell Mol Life Sci 59:882-893

28. Das GD, Pfaffenroth MJ 1977 Experimental studies on the postnatal development of the brain: III. Cerebellar development following localized administration of ENU. Neuropath Appl Neurobiol 3:191-212

29. Darmanto W, Inouye M, Hayasaka S, Takagishi Y, Aolad HM, Murata Y 1998 Dose response relationship of disturbed migration of Purkinje cells in the cerebellum due to X-irradiation. Environ Med 42:46-50

30. Badaeva LN 1981 Experimental study of the postnatal neurotoxic effect of chloroorganic pesticides. Folia Morphol (Praha) 29:113-114 